\title{
Implications of Ice Morphology for Comet Formation
}

\author{
M. P. Collings, J. W. Dever, M. R. S. McCoustra \\ School of Chemistry, University of Nottingham, University Park, \\ Nottingham, U.K., NG7 $2 R D$.
}

\section{H. J. Fraser}

Raymond and Beverly Sackler Laboratory of Astrophysics, Leiden Observatory, University of Leiden, Niels Bohr Weg 2, 2300 RA, Leiden, Netherlands.

\begin{abstract}
Laboratory surface science under ultra-high vacuum (UHV) conditions allows us to simulate the growth of ices in astrophysical environments. Using the techniques of temperature programmed desorption (TPD), reflectionabsorption infrared spectroscopy (RAIRS) and micro-balance methods, we have studied binary ice systems consisting of water $\left(\mathrm{H}_{2} \mathrm{O}\right)$ and variety of other species including carbon monoxide $(\mathrm{CO})$, at astrophysically relevant conditions of temperature and pressure. We present results that demonstrate that the morphology of water ice has an important influence on the behavior of such systems, by allowing processes such as diffusion and trapping that can not be understood through a knowledge of the binding energies of the species alone. Through an understanding of the implications of water ice morphology on the behavior of ice mixtures in the interstellar environment, additional constraints can be placed on the thermodynamic conditions and ice compositions during comet formation.
\end{abstract}

A commonly applied description of the structure of the ice mantles accreted on interstellar dust grains is the 'onion layered' model (Allamandola et al 1999, Ehrenfreund et al 1998). In this model, the dust grain is surrounded by an inner layer of hydrogenated (polar) ice, which in turn is coated with an outer layer of non-hydrogenated (apolar) ice. Since the composition of each layer is dominated, respectively, by water $\left(\mathrm{H}_{2} \mathrm{O}\right)$ and carbon monoxide $(\mathrm{CO})$, our laboratory representation of this model is a film of $\mathrm{H}_{2} \mathrm{O}$ upon which a film of $\mathrm{CO}$ is subsequently deposited.

We have demonstrated previously that the desorption characteristics of CO are strongly dependent on the morphology of the underlying water film (Collings et al 2003a, b). As the water ice undergoes an irreversible phase change from a high density to a lower density amorphous structure during thermal processing, $\mathrm{CO}$ molecules become trapped within pores as pathways to the surface are sealed off. Such $\mathrm{CO}$ entrapment can occur even when the $\mathrm{CO}$ is initially in a separate layer, since the molecules in the solid $\mathrm{CO}$ film become mobile and diffuse into the porous water film at less than $15 \mathrm{~K}$, well below the temperature at which the sublimation of solid CO becomes significant. Trapped CO is released as the water film crystallizes and again as the water desorbs.

Using a stochiastic integration software package, we have developed a kinetic simulation incorporating the phase changes of the water ice and diffusion of $\mathrm{CO}$ to accurately reproduce the complex desorption traces of the $\mathrm{CO} / \mathrm{H}_{2} \mathrm{O}$ system 
in laboratory experiments (Collings et al 2003b). The simulation can then be adapted to suit astrophysical time scales, and hence provide a prediction of the desorption behavior of ice mantles during the formation of the pre-solar nebula. Current models of desorption in the pre-solar nebula tend to treat the nonhydrogenated layer in isolation, allowing it to sublime entirely at roughly $20^{\circ} \mathrm{K}$. The results displayed in figure 1 predict that (at the film thickness simulated) some 15 to $25 \%$ of the $\mathrm{CO}$ in the outer layer will become trapped in the low density amorphous ice. This portion of $\mathrm{CO}$ will be retained in any amorphous water ice that is subsequently incorporated in cometary material, and is available for chemical reaction in the warm ice.

The kinetic parameters for the desorption processes, determined through a combination of laboratory experiment and simulation, are also of great worth in the analysis of other significant scenarios, for example, condensation in the solar nebula. The condensation coefficient, $\alpha$ (fraction of incident molecules condensed), is given by

$$
\alpha=S-R / Z_{W},
$$

where $S$ is the sticking probability, $R$ is the rate of desorption of the species, and $Z_{W}$ is the rate at which gas phase molecules of the species impinge on the surface, which is a function of temperature and pressure. For $\mathrm{CO}$ impinging on a water ice surface at low temperature, it is reasonable to assume that the sticking probability is unity. Figure 2 shows a plot of the gas phase pressure against temperature at which $\alpha$ is zero, for a condensation of amorphous and crystalline water ice, solid $\mathrm{CO}$, and $5 \% \mathrm{CO}$ in water ice, which have been calculated using desorption kinetics that we have measured by experimentation and simulation (Fraser et al 2001, Collings et al 2003b). These curves effectively mark the 'vapor pressure' for the process - condensation occurs above (and to the right) of the curve. These plots demonstrate that at realistic pressures, the condensation of $\mathrm{CO}$ can only occur at temperatures below about $40 \mathrm{~K}$, below the temperatures typically quoted for comet formation.

In conclusion, the morphology of water ice, through the entrapment of volatile species such as $\mathrm{CO}$ within its porous structure, is a highly significant factor influencing the composition of interstellar ices incorporated into comets. An understanding of the kinetics of desorption processes provides a link between experiments performed under laboratory conditions and the behavior of ices in astrophysical environments.

Acknowledgments. This research was performed with the financial support of Particle Physics and Astronomy Research Council (PPARC) U.K., the Leverhulme Trust and the University of Nottingham. The authors gratefully acknowledge the advice of David A. Williams and Serena Viti. MPC thanks the Sir Eric Rideal Trust and the IAU Travel Bursaries for support in attending this meeting.

\section{References}

Allamandola, L. J., Bernstein, M. P., Sandford, S. A., \& Walker, R. L. 1999, Space Sci.Rev., 90,219

Collings, M. P., Dever, J. W., Fraser, H. J., McCoustra, M. R. S., \& Williams, D. A. 2003a, ApJ, 583, 1058 


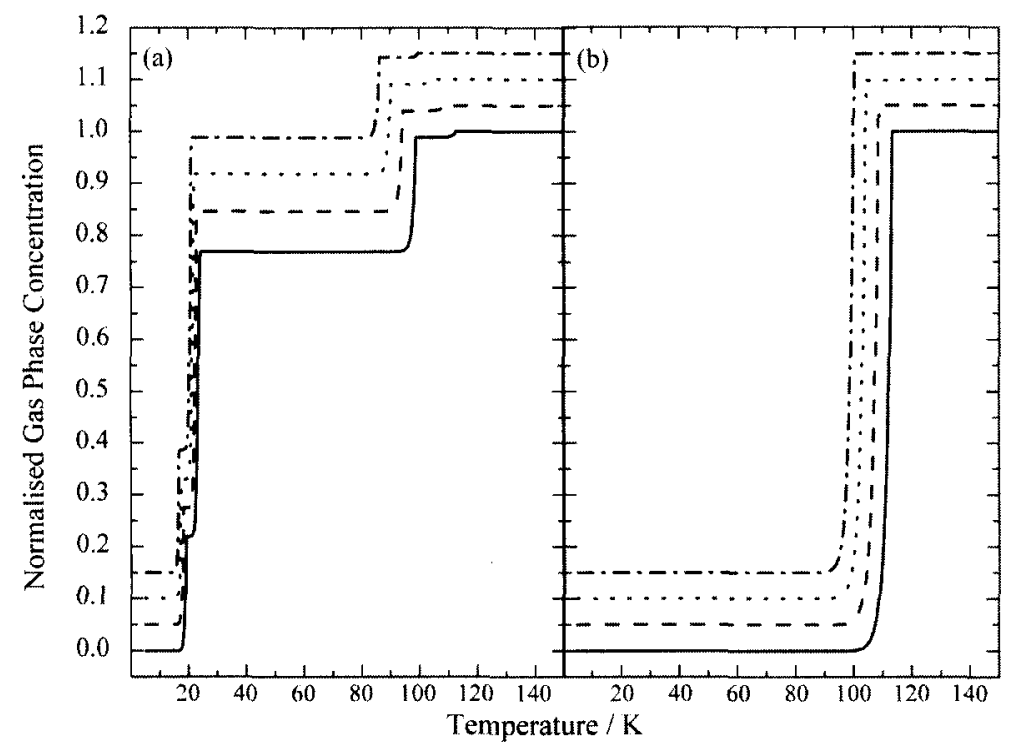

Figure 1. Normalized gas phase concentrations (offset for clarity) of (a) $\mathrm{CO}$, and (b) $\mathrm{H}_{2} \mathrm{O}$, as a function of temperature as predicted by a kinetic simulation for heating rates of $1 \mathrm{~K}_{\text {year }}{ }^{-1}$ (solid), $1 \mathrm{~K}$ decade $^{-1}$ (dashed), 1 $\mathrm{K}$ century $^{-1}$ (dotted) and $1 \mathrm{~K}$ millenium $^{-1}$ (dot-dashed). 


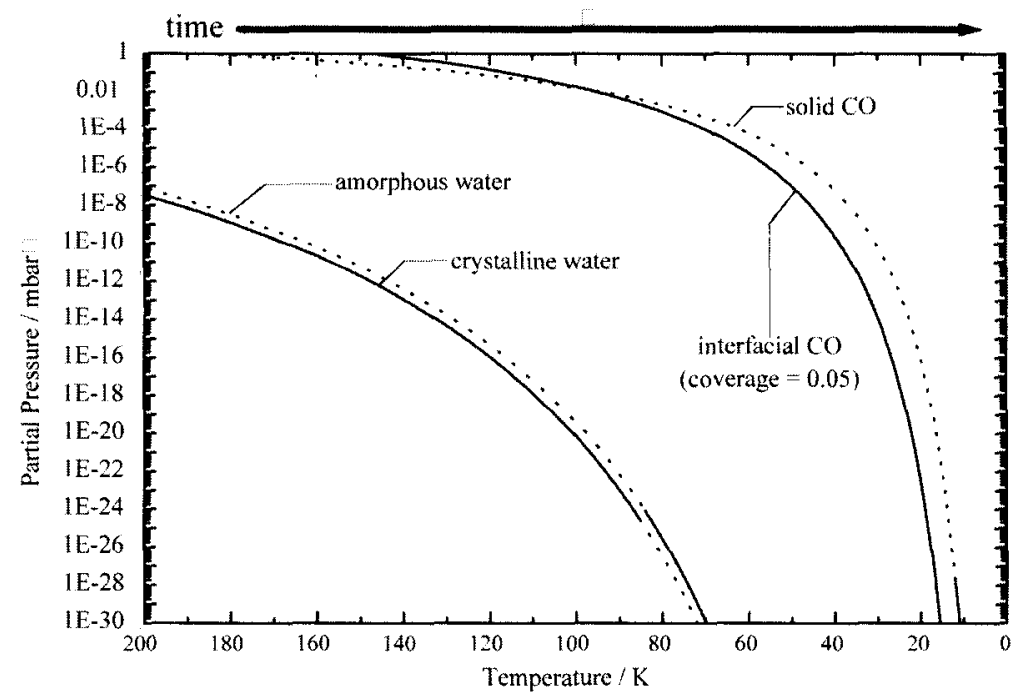

Figure 2. Plots of the pressure and temperature conditions for which condensation $=$ desorption for amorphous and crystalline water ice, solid $\mathrm{CO}$ ice, and $\mathrm{CO}$ adsorbed with a coverage of 0.05 on water ice (gas phase temperature and dust temperature are assumed to be equal). Dotted curves show where certain species can not be formed; i.e., water ice is crystalline when condensed above $\sim 80 \mathrm{~K}$ and amorphous when condensed below $80 \mathrm{~K}$; solid $\mathrm{CO}$ is mobile above $\sim 15 \mathrm{~K}$ and will diffuse into the porous water ice.

Collings, M. P., Dever, J. W., Fraser, H. J., \& McCoustra, M. R. S. 2003b, A\&SS, 285, 1

Ehrenfreund, P., Dartois, E., Demyk, K, \& d'Hendecourt, L. 1998, A\&A, 339, L19

Fraser, H. J., Collings, M. P., McCoustra, M. R. S., \& Williams, D. A. 2001, MNRAS, 327,1165 